\title{
EXPERIMENTAL AND THEORETICAL SPECTROSCOPIC STUDIES ON SELECTED IGEPALS
}

\author{
G. Pasterna ${ }^{a}$, T. Kupka ${ }^{a}$, R. Wrzalik ${ }^{a}$, K. Pasterny ${ }^{a}$ \\ AND J.S. BLICHARSKI ${ }^{b}$ \\ ${ }^{a}$ Institute of Physics, University of Silesia, Uniwersytecka 4, 40-007 Katowice, Poland \\ ${ }^{b}$ Institute of Physics, Jagiellonian University, Reymonta 4, 30-086 Kraków, Poland
}

Several nonionic igepals characterized by the formula $\left(\mathrm{C}_{m} \mathrm{H}_{2 m+1}\right)-\mathrm{C}_{6} \mathrm{H}_{4}$ $\left(\mathrm{OCH}_{2} \mathrm{CH}_{2}\right)_{n} \mathrm{OH}$ were investigated by high resolution $\mathrm{NMR}$ and IR spectroscopy. Gauge invariant atomic orbital density functional theory NMR calculations on model molecules in the gas phase additionally supported the assignment of experimental carbon signals. Different orientations of individual sub-units of an aliphatic chain relative to external magnetic field and trans-gauche transitions were assumed to explain complex patterns of carbon spectra in the aliphatic region of igepals with linear alkyl chain.

PACS numbers: 32.30.Dx, 33.20.Ea, 31.15.Ar

\section{Introduction}

Igepals are typical examples of surfactants. Surfactants occur widely in nature and they have been used for over 1000 years in everyday application as emulsifiers in cleaning and in foods. Igepals are known to exist in water in several ordered phases (lyotropic liquid crystals) depending on amphiphilic concentration and temperature. The structure and properties of lyotropic liquid crystalline phases are of great interest because they seem to play an important role in many biological systems including cell membranes [1]. Igepal molecules are formed of two main parts, a hydrophobic alkyl chain, linear or branched, and a hydrophilic polyoxyethylenic chain terminated by a polar $\mathrm{OH}$ group. Both chains are bridged by phenyl ring (para-substitution). Such a structure is typical of many detergents and surfactants.

In this work we report on NMR studies, complemented by IR measurements, of selected igepal characterized by the formula $\left(\mathrm{C}_{m} \mathrm{H}_{2 m+1}\right)-\mathrm{C}_{6} \mathrm{H}_{4}-\left(\mathrm{OCH}_{2} \mathrm{CH}_{2}\right)_{n} \mathrm{OH}$, namely, igepal CA-520 ( $m=8, n=5$, polyoxyethylene(5) isooctylphenyl ether), igepal CA-720 ( $m=8, n=12$, polyoxyethylene(12) isooctylphenyl ether), igepal CO-520 ( $m=9, n=5$, polyoxyethylene(5) nonylphenyl ether), and igepal CO-720 ( $m=9, n=12$, polyoxyethylene(12) nonylphenyl ether). 


\section{Results and discussion}

The $75 \mathrm{MHz}$ high resolution carbon NMR spectra were recorded for a few igepal samples using VARIAN VXR-300 NMR spectrometer. All igepals were purchased from Aldrich. Figures 1 and 2 show the proton decoupled ${ }^{13} \mathrm{C}$ spectra of CA-520 and CO-520, respectively. Because of the limited scope of this report we do not present spectra of CA-720 and CO-720. The observed lines are fairly narrow and some of them are well resolved for all igepals under consideration. However, the number of signals for igepal CO-520 (and CO-720) is significantly higher than the number of carbon atoms in this molecule. In particular the aliphatic spectral region is overcrowded.

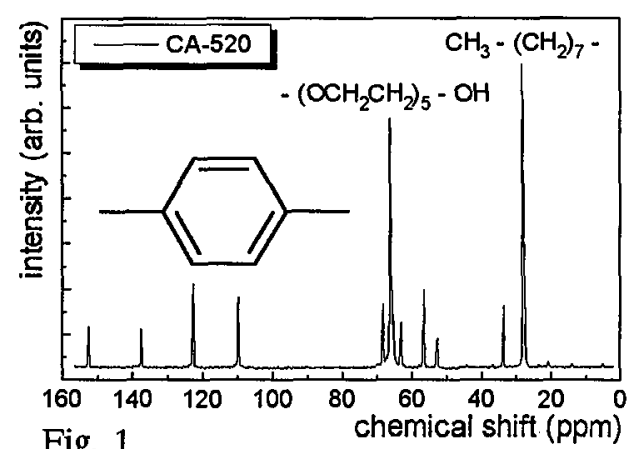

Fig. 1



Fig. 2

Fig. 1. ${ }^{13} \mathrm{C}$ NMR spectrum of igepal CA-520.

Fig. 2. ${ }^{13} \mathrm{C}$ NMR spectrum of igepal CO-520.

A fast rotational motion of CO-520 molecule should average the positions of individual carbon lines. Thus, no more than 9 signals should be seen in the aliphatic region of the spectrum. The aliphatic chain that contains nine carbon atoms is long enough to wind itself round the core of CO-520 molecule, somehow. Therefore, different orientations of $\mathrm{C}-\mathrm{H}$ bonds relative to external magnetic field are possible. This results in appearance of many resonances for every carbon atom [2]. On the other hand, it is known that the change from trans to gauche conformations causes downfield shift of carbon lines in the range from 1.4 to $4.1 \mathrm{ppm}$ [3]. Therefore, the presence of many trans-gauche conformers produces very complex spectra. In addition, a mobility of alkyl chain is significantly lower than the oxyethylenic one in compounds structurally related to igepals [4]. The net result of the mentioned above processes, which are slow on the NMR time scale, is a very complex ${ }^{13} \mathrm{C}$ proton decoupled spectrum of igepals CO-520 and CO-720.

To support the above considerations as well as assignment of experimental carbon signals gauge invariant atomic orbital (GIAO) - density functional theory (DFT) NMR calculations [5] on model molecules in the gas phase were carried out. Due to the size of igepal CO-520 molecule calculations were started with fast molecular modelling MM+ optimization and later semi-empirical PM3 
minimization geometry was performed using HYPERCHEM v. 4.5 software. The optimized PM3 geometry of linear molecule was used as input for GIAO NMR calculations. In the next step several molecules with deformed linear alkyl chain were constructed by rotation of $180^{\circ}$ on a particular C-C bond. A small 3-21G basis set was used in single point NMR calculations employing a recent B3PW91 density functional. In addition, GIAO NMR shieldings of benzene and methane were calculated. These molecules were selected as references, in order to recalculate theoretical isotropic shieldings to chemical shifts for aromatic and non-aromatic parts of igepal CO-520, respectively. In theoretical calculations of nuclear shieldings only one conformation of the chain substituent relative to the aromatic ring was considered. Individual carbon chemical shifts, calculated for linear and several deformed molecules of igepal CO-520, are shown in Fig. 3. Theoretical spectra consist of three groups of signals, namely, aromatic, oxyethylenic, and aliphatic ones. Values of theoretical shieldings (chemical shifts) are strongly dependent on aliphatic chain shape. This effect decreases with distance from perturbation site. Nevertheless, it is also observed for aromatic signals. In contrary, the oxyethylenic signals are almost non-perturbed. A dispersion of aliphatic signals predicted by the theory was observed, so, due to presence of different alkyl chain conformation, unusual pattern of experimental spectrum appeared. The model discussed above reproduces qualitatively the experimental carbon spectra for CO-type igepals. In the case of CA-type igepals the branched alkyl chain cannot be deformed in such a way as linear chain in CO-type ones and in consequence one observes much less complicated, typical [4], spectra for CA-520 and CA-720 samples.



Fig. 3

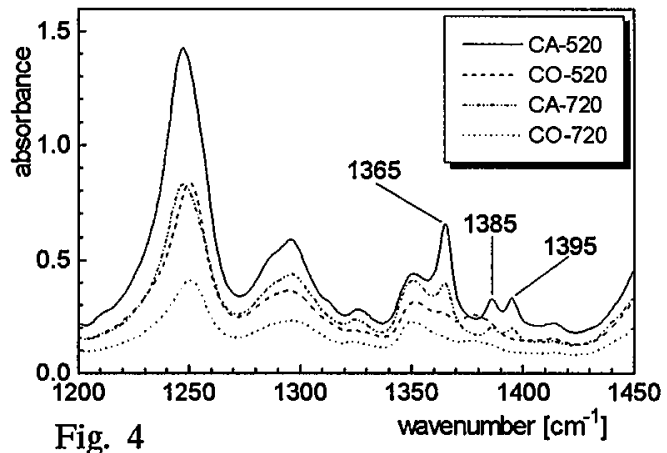

Fig. 4

Fig. 3. Theoretical chemical shifts for linear and deformed molecules of igepal CO-520. The numbers on the vertical axis denote the carbons in the $\mathrm{C}-\mathrm{C}$ bond around which the rotation has been performed to obtain deformed alkyl chain. The numbering starts from the carbon in the $\mathrm{CH}_{3}$ group.

Fig. 4. FT-IR spectra of neat igepals in the frequency range $1200-1450 \mathrm{~cm}^{-1}$.

NMR studies have been supplemented by IR measurements which were performed for neat igepals and their solutions in $\mathrm{CCl}_{4}$ using Bio-Rad FTS6000 spectrometer. The obtained FT-IR spectra for all studied neat igepals as well as their 
solutions in carbon tetrachloride exhibit very similar structure. The differences connected with different structure of alkyl chains (branched or linear) appear in the frequency range of $1200-1450 \mathrm{~cm}^{-1}$, see Fig. 4, where the bands associated with deformation vibrations of $\mathrm{CH}_{3}$ and $\mathrm{CH}_{2}$ groups occur. In the case of CA-type igepals a significant increase in the intensity of the deformation $\mathrm{CH}_{3}$ modes $\left(1365 \mathrm{~cm}^{-1}\right)$ relatively to $\mathrm{CH}_{2}$ modes is observed. Additionally, $1385 \mathrm{~cm}^{-1}$ and $1395 \mathrm{~cm}^{-1}$ modes corresponding to deformation vibrations of $\mathrm{CH}_{3}$ groups emerge for this type of igepals. The presence of these bands and increase in their intensity reflects the branched structure of the alkyl chains in CA-igepals.

\section{References}

[1] C.E. Fairhurst, S. Fuller, J. Gray, M.C. Holmes, G.J.T. Tiddy, in: Handbook of Liquid Crystals, Vol. 3, Eds. D. Demus, J. Goodby, G.W. Gray, H.-W. Spiess, V. Vill, Wiley-VCH, Weinheim 1998, p. 341.

[2] A. Losche, Bull. Magn. Reson. 3, 109 (1978).

[3] T. Kato, T. Uryu, Mol. Cryst. Liq. Cryst. 195, 1 (1991).

[4] A.A. Ribeiro, E.A. Dennis, J. Phys. Chem. 80, 1746 (1976).

[5] M.J. Frisch, A. Frisch, J.B. Foresman, GA USSIAN 94, Users Reference, Gaussian Inc., Pittsburgh (PA) 1995. 